\title{
BioBran-augmented maturation of human monocyte-derived dendritic cells
}

\author{
D. CHOLUJOVA, J. JAKUBIKOVA, J. SEDLAK*
}

Laboratory of Tumor Immunology, Cancer Research Institute, Slovak Academy of Sciences, Bratislava, Slovak Republic, e-mail: exonsedl@savba.sk

\section{Received September 17, 2008}

\begin{abstract}
BioBran, enzymatically modified arabinoxylan from rice bran was tested for its possible effects on in vitro maturation of human dendritic cells (DC). Immature DC (iDC) derived from plastic-adhered, IL-4 and GM-CSF treated peripheral monocytes (Mo) were further cultured with cytokine maturation mix 1 (CMM1; TNF- $\alpha$, IL-1 $\beta$ and IL-6) or CMM2 (LPS and IFN- $\gamma$ ) to induce their maturation into mature DC (matDC1 or matDC2, respectively). Different concentrations of BioBran (10, 100, 400 and $1000 \mu \mathrm{g} / \mathrm{ml}$ ) were applied in the presence or absence of relevant CMM to assess the effects of BioBran on DC maturation processes. BioBran induced maturation of iDC, as these cells cultured with IL-4/GM-CSF/BioBran down-regulated CD14 and CD1a antigens on cell surface and significantly increased expression of maturation marker CD83. The increase of surface density of costimulatory molecules CD80 and CD86 on iDC in the presence of BioBran was also observed. In addition, BioBran induced functional maturation of iDC, confirmed by decreased endocytic activity of iDC. Furtheremore, BioBran enhanced maturation potential of cytokine mixes, as both matDC1 and matDC2 exposed to BioBran completely lost CD14 and upregulated CD83, CD80 and CD86 antigens, in comparison to DC matured with the relevant CMM alone. BioBran also increased CD123 antigen expression on all DC subsets. Interestingly, matDC2 matured in the presence of BioBran $(400 \mu \mathrm{g} / \mathrm{ml})$ expressed higher levels of CD123 and lower levels of CD11c cell surface antigens, the phenotype represented by CD11 $\mathrm{c}^{\text {dim }}$ CD123 bright plasmacytoid DC population. These data demonstrate that BioBran is a potent enhancer of DC maturation and suggest that BioBran might be a useful agent to create the environment that favours DC maturation.
\end{abstract}

Key words: Dendritic cell, maturation, BioBran, buffy coat

Dendritic cells are professional antigen-presenting cells (APC) that play the central role in initiation and regulation of immune responses since they control both the innate (NK cells, NKT cells, $\gamma \delta$ Tcells) and the adaptive (T and B cells) arm of immunity [1]. DC include subsets of cells with different characteristics that are derived from bone marrow progenitor cells. In humans, myeloid and lymphoid precursors can give rise to $\mathrm{CD} 11 \mathrm{c}^{\text {bright }} \mathrm{CD} 123^{\mathrm{dim}}$ myeloid dendritic cells ( $\mathrm{mDC}$ ) or CD11 $\mathrm{c}^{\mathrm{dim}} \mathrm{CD} 123^{\text {bright }}$ plasmacytoid dendritic cells (pDC), respectively [2]. Peripheral tissue antigen-loaded DC migrate through the afferent lymphatics into the draining lymph nodes where they present processed protein and lipid antigens to T cells via both classical (major histocompatibility complex (MHC) class I and class II) and non-classical antigen-presenting molecules. Nonactivated iDC present antigens to $T$ cells which in the absence of appropriate costimulation leads to tolerance $[3,4]$. Upon encounter with pathogens or other 'danger'-associated stimuli, such as cytokines, products of dam-

\footnotetext{
* Corresponding author
}

aged tissues or innate lymphocytes, DC undergo a process of activation and maturation, wherein they loose ability to capture $\mathrm{Ag}$, but acquire the capacity to activate immunity. This process of maturation includes changes in morphology and motility, translocation of MHC to the cell surface, increased expression of costimulatory molecules (CD80/CD86 and CD40), and production of cytokines that determine the class of immune response by selectively polarizing the development of effector T cells [5].

Due to their ability to initiate, link and regulate both innate and adaptive immunity, DC represent a potential tool for cancer immunotherapy. Both $\mathrm{mDC}$ and $\mathrm{pDC}$ were found to infiltrate several types of human tumors but most of the tumor-infiltrating DC were immature DC [6]. It was also shown that several tumorderived factors lead to inhibition of DC maturation, including IL-10, VEGF, TGF- $\beta$, and PGE2 [7]. DC encountered by tumor antigen in the absence of pro-maturation environment remains in immature or semi-mature state and a tolerogenic response may be generated [4]. Therefore, promoting DC maturation was proposed as a therapeutic objective to counter tumor-driven tolerance and stimulate anti-tumor immunity. 
BioBran is a food supplement which is obtained by reacting rice bran hemicellulose with multiple carbohydrate hydrolyzing enzymes from the Shiitake mushrooms. The active component of BioBran is an arabinoxylan. Previous research has suggested that the product enhances natural killer (NK) cell activity in vitro and in vivo $[8,9]$. The objective of this study was to investigate the possible modulatory effects of BioBran on in vitro differentiation and maturation of human DC.

\section{Materials and methods}

Reagents. The following monoclonal antibodies (mAbs) were used in this study: fluorescein isothiocyanate (FITC)-conjugated anti-human CD11c (IgG1) mouse mAb, phycoerythrin (PE)conjugated anti-human CD14 (IgG1) mouse mAb, PE anti-human CD45 (IgG1) mouse mAb, PE anti-human CD123 (IgG1) mouse mAb, PE anti-human CD80 (IgG1) mouse mAb, PE anti-human CD83 (IgG2bא) mouse mAb, PE anti-human

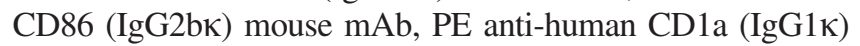
mouse $\mathrm{mAb}$, and energy-coupled dye (ECD) anti-human HLADR (IgG1) mouse mAb. All mAbs and isotype-matched controls were purchased from Immunotech Beckman Coulter. We also used the following cytokines and ligands: recombinant human granulocyte-macrophage colony-stimulating factor (rhuGM-CSF; Santa Cruz Biotechnology), recombinant human interleukin-4 (rhuIL-4; Santa Cruz), recombinant human interleukin-1 $\beta$ (IL-1 $\beta$; Biomol), recombinant human interleukin-6 (IL-6; Santa Cruz), tumor necrosis factor (TNF$\alpha$; Santa Cruz), lipopolysacharide (LPS; Sigma-Aldrich), and interferon- $\gamma$ (IFN- $\gamma$; Bender MedSystem).

BioBran - denaturated hemicellulose obtained from rice bran that is treated enzymatically with an extract from Shiitake mushrooms (Lentinus edodes). The main chemical structure and an active component is an arabinoxylan with a xylose in its main chain and an arabinose polymer in its side chain. Biobran was provided by Daiwa Pharmaceuticals Co. Ltd, Tokyo, Japan. BioBran stock solution was prepared by dissolving 1 dose of BioBran 1000 in distilled $\mathrm{H} 2 \mathrm{O}$ at final concentration of $10 \mathrm{mg} / \mathrm{ml}$.

Isolation of peripheral blood monocytes. Monocytes were isolated from buffy coats of healthy donors (National Transfusion Service, Bratislava) by Pancol density gradient centrifugation (1.077 g/ml, PAN-Biotech, Germany). The mononuclear cells from the interface were collected, washed twice with PBS and once in AIM-V complete medium (GibcoBRL, Paisley, UK), and then allowed to adhere in the plastic 6-well plates for 2 hours at $37{ }^{\circ} \mathrm{C}, 5 \% \mathrm{CO}_{2}$. The nonadherent cells were gently removed by washing three times with PBS and once with AIM-V medium.

Dendritic cell differentiation and maturation. Plastic-adhered monocytes were cultured in 6-well plates for 6 days in AIM-V complete medium supplemented with GM-CSF (1000 IU/ml) and IL-4 (1000 IU/ml) to generate iDC. The fresh medium with cytokines was added to cell cultures every second day. On day 7 the medium was exchanged and cell were cultured in com- plete AIM-V medium containing GM-CSF $(1,000 \mathrm{U} / \mathrm{mL})$ and IL-4 $(1,000 \mathrm{U} / \mathrm{mL})$ in the presence or absence of maturation stimuli for a further 2 days. Cytokine maturation mix 1 (CMM1; TNF- $\alpha$ (10 ng/ml), IL-1 $\beta$ (10 ng/ml) and IL-6 (10 ng/ml)), and cytokine maturation mix 2 (CMM2; LPS (250 ng/ml) and IFN$\gamma(1000 \mathrm{IU} / \mathrm{ml}))$ with or without addition of BioBran $(10,100$, 400 and $1000 \mu \mathrm{g} / \mathrm{ml}$ ) were used as stimuli for maturation of DC into matDC1 and matDC2, respectively [10].

Immunophenotypic analysis of cells. Monocyte-derived DC were collected, washed and resuspended in PBS $0.2 \%$ BSA

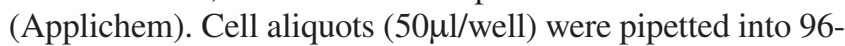
well V-bottomed microplate and incubated with $2 \mu \mathrm{l}$ of relevant fluorochrome-conjugated $\mathrm{mAbs}$ for $30 \mathrm{~min}$ at $37 \mathrm{oC}$ in the dark. At the end of incubation, samples were transfered into cytometric test tubes and $2 \mu \mathrm{l}$ of 7 -amino-actinomycin D (7-AAD; stock solution $1 \mathrm{mg} / \mathrm{ml}$ in PBS) in $300 \mu$ of PBS were added to label nonviable cells. Samples were incubated on ice for $10 \mathrm{~min}$, protected from light and analysed using a Coulter Epics Altra flow cytometer.

Endocytosis assay with FITC-dextran. The efficiency of endocytosis was evaluated as cellular uptake of fluorescein isothiocyanate (FITC)-conjugated dextran by monocytes, immature and mature DC (matDC1, matDC2) cultured in the absence or presence of different concentrations of BioBran (100, 400 and $1000 \mu \mathrm{g} / \mathrm{ml}$ ). For the endocytosis assay, 2 x $10^{5}$ cells per sample were incubated in AIM-V medium with $1 \mathrm{mg} / \mathrm{ml}$ of FITC-dextran ( $\mathrm{Mr} 40,000$; Sigma) for $60 \mathrm{~min}$ either at $37^{\circ} \mathrm{C}$ (for uptake) or $4{ }^{\circ} \mathrm{C}$ (negative control). After incubation cells were washed three times with ice-cold PBS. Additionally, trypan blue $(0.2 \%)$ was used for quenching surface-bound FITC fluorescence. The accumulation of FITC-dextran within the cells was quantitated by flow cytometry. Nonviable cells and contaminating lymphocytes were excluded according to their scatter properties and 7-AAD staining. The values were calculated as a change in percentage of green fluorescence positivity between cell samples incubated at $37{ }^{\circ} \mathrm{C}$ and $4{ }^{\circ} \mathrm{C}$, respectively.

Flow cytometric analysis. Samples were analyzed using an Epics Altra (Beckman Coulter) four-colour flow cytometer equipped with an argon laser $(15 \mathrm{mV})$ source operating at 488 $\mathrm{nm}$. The emission of fluorochromes was recorded through specific band-pass fluorescence filters: FITC, $525 \mathrm{~nm}$ (FL1); PE, 575 nm (FL2); ECD, 610 nm (FL3); 7-AAD; 675 nm (FL4). Data were analysed using WinMDI version 2.7 software (J. Trotter, Scripps Research Institute, La Jolla, CA). For immunophenotyping, gating was done on side scatter (SSC; ordinate) versus log-scale orange fluorescence of ECD dye (HLA-DR positivity; abscissa), to separate residual lymphocytes. Nonviable (7-AAD-positive) cells were excluded from analysis. An average of 20000 cells were collected per sample.

\section{Results}

Phenotypic characterization of Mo and Mo-derived DC at different stages of in vitro maturation. The phenotypic analysis was performed to confirm the differentiation and 

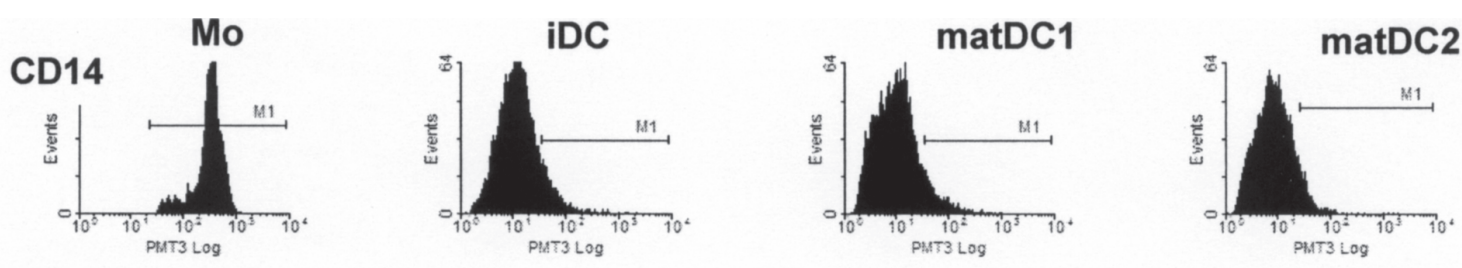

\section{HLA-DR}
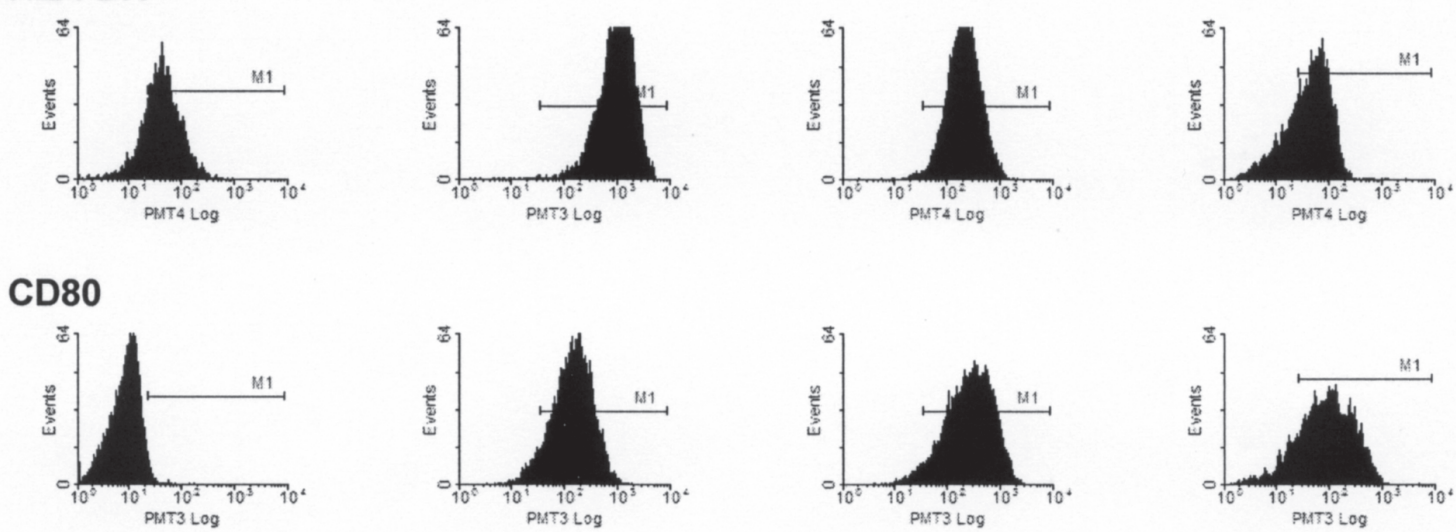

\section{CD83}
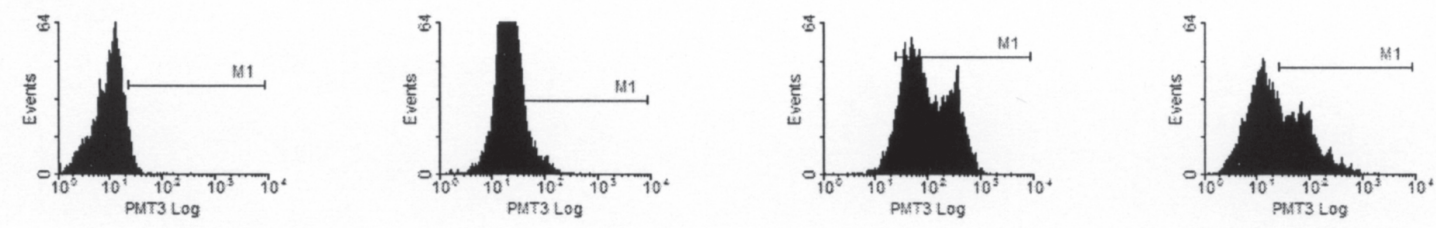

\section{CD86}
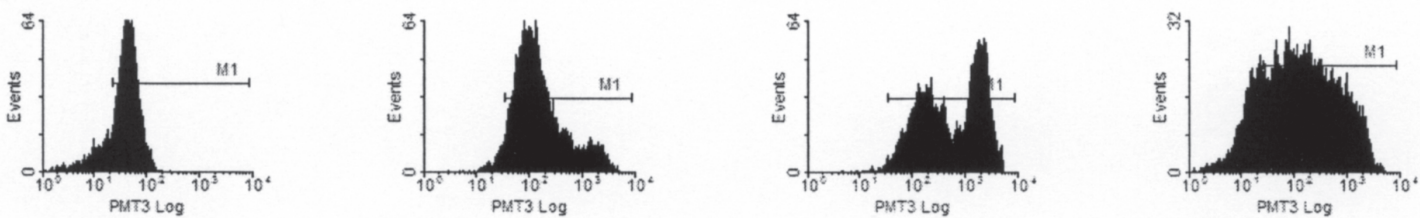

\section{CD1a}
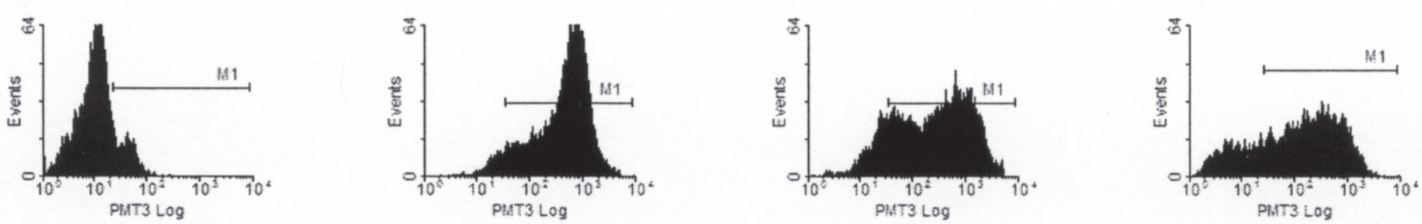

Figure 1. The phenotype of Mo and DC at different stages of in vitro maturation. Human peripheral Mo were analyzed by flow cytometry and were cultured with GM-CSF and IL-4 (1000 UI/ml) for 6 days to obtain iDC. The iDC were cultured in the presence of GM-CSF and IL-4 for additional 2 days either with CMM1 or CMM2 to induce their maturation into matDC1 and matDC2, respectively. At the end, iDC, matDC1 and matDC2 subsets were harvested, stained with fluorochrome-conjugated mAbs against the indicated cell surface antigens, and analyzed using flow cytometry. This presented phenotype of Mo and DC is typical of more than five cell cultivations.

maturation status of DC. Human peripheral CD14+ Mo were isolated by plastic adherence from buffy coats of healhy donors and cultured in the presence of GM-CSF and IL-4 for 6 days to induce their differentiation into iDC. Additional 2- day treatment of iDC with two different maturation cytokine cocktails (CMM1, CMM2) was used to obtain 2 distinct populations of mature DC, termed matDC1 and matDC2. As shown in Fig. 1, Mo prominently expressed CD14 and HLA-DR. They 

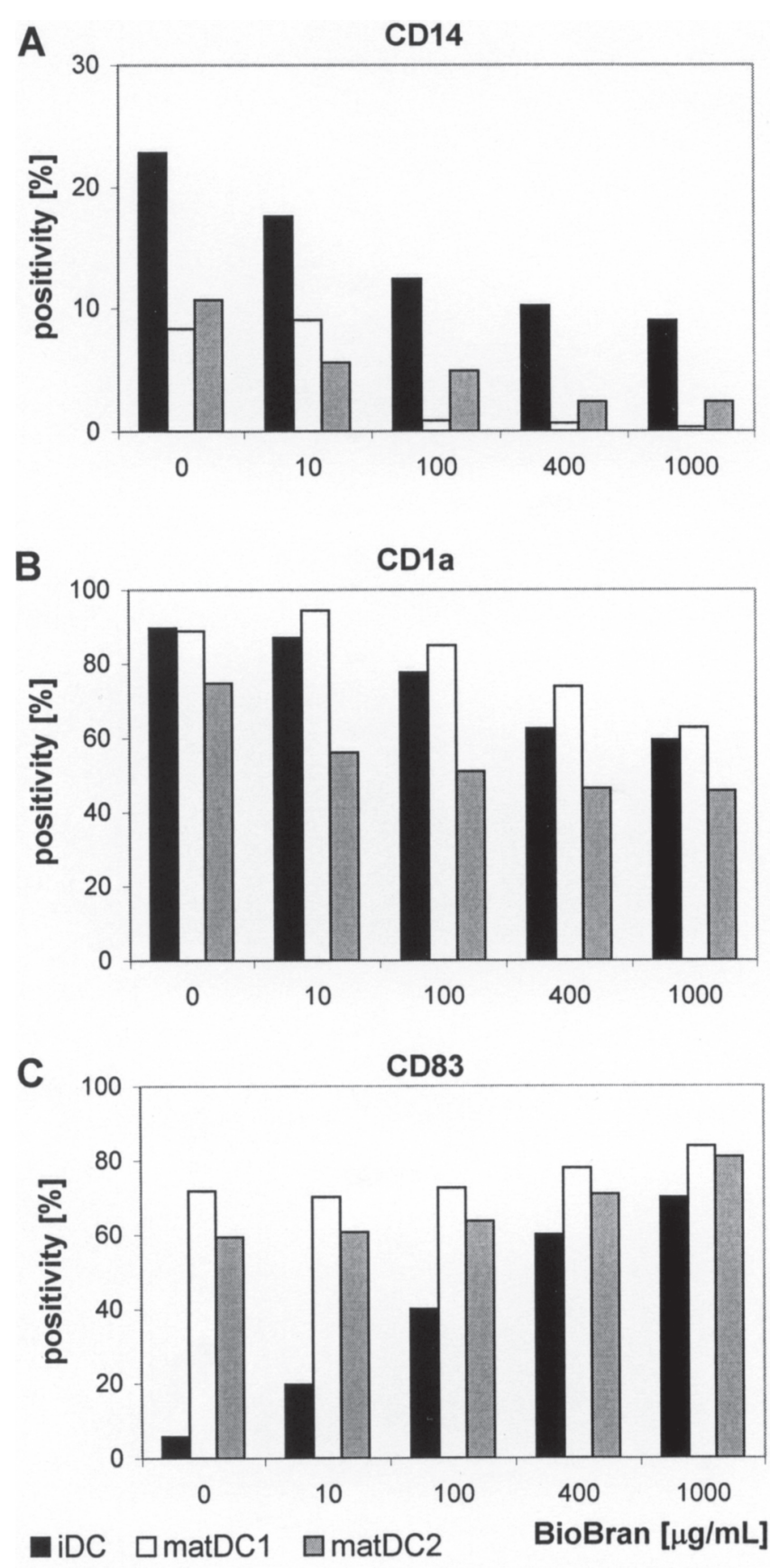

Figure 2. The effect of BioBran on the expression of differentiation/ maturation antigens on DC subsets. The surface expression of CD14, CD1a and DC maturation marker CD83 was examined by flow cytometry on HLA-DR positively gated iDC, matDC1 and matDC2. The percentage of cell positivity is shown and is representative of three independent experiments.

also showed low CD86 positivity, but almost completely lacked the expression of CD1a, CD83 and CD80. In contrast to Mo, iDC down-regulated CD14, but strongly expressed antigen-presenting molecule CD1a. They were positive for

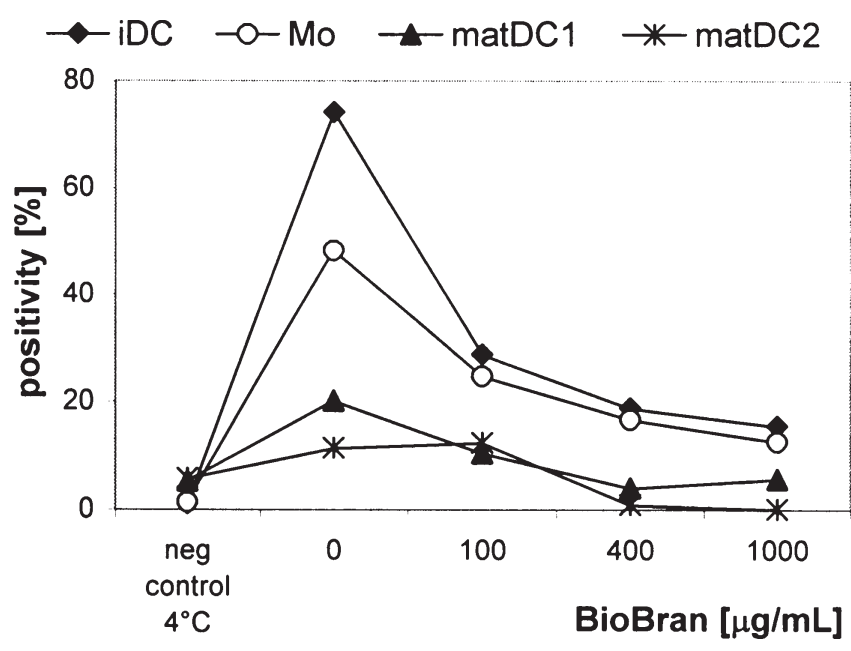

Figure 3. Endocytic activity of Mo and DC populations. Cells were incubated with FITC-labeled dextran $(1 \mathrm{mg} / \mathrm{ml})$ for $60 \mathrm{~min}$ at $37{ }^{\circ} \mathrm{C}$ and $4{ }^{\circ} \mathrm{C}$ (negative control). Trypan blue was used for quenching of surfacebound green fluorescence. Endocytosis was assessed by flow cytometry as the percentage of dextran positive cells. Results shown are representative from three similar experiments performed on cells from different donors.

expression of costimulatory molecules CD80 and CD86, and showed marginal CD83 expression. Mature DC (both matDC1 and matDC2), extensively increased the expression of maturation marker CD83 and costimulatory molecules CD80 (B7-1) and CD86 (B7-2).

BioBran modulates expression of differentiation and maturation markers on DC. To study the effect of BioBran on maturation of DC, iDC were treated with different concentration $(10,100,400$ and $1000 \mu \mathrm{g} / \mathrm{ml})$ of BioBran in the presence or absence of relevant maturation mix (Fig. 2). The surface expression of CD14, CD1a and CD83 antigens on iDC, matDC1 and matDC 2 was examined by flow cytometry on HLA-DR+ gated cells. BioBran down-regulated the expression of monocyte marker CD14 (Fig. 2A) and antigen-presenting molecule CD1a (Fig. 2B) on the surface of iDC, but extensively increased the expression of CD83 (Fig. 2C), a marker of mature DC. Similar to iDC, down-regulation of CD14 and CD1a and upregulation of CD83 were observed in both mature DC populations (matDC1 and matDC2) with increasing concentrations of BioBran used.

BioBran decreases endocytic activity of iDC. BioBran was tested for its ability to affect the endocytic activity of Mo and Mo-derived DC. Endocytosis was evaluated by the uptake of FITC-conjugated dextran at $37^{\circ} \mathrm{C}$, with negative control incubated at $4{ }^{\circ} \mathrm{C}$. The untreated iDC and Mo showed high level of endocytic activity (73\% and $48 \%$ of FITC-dextran-positive cells, respectively), whereas both matDC1 and matDC2 showed clear reduction of dextran-FITC uptake $(14.9 \%$ and $5.6 \%$, respectively; Fig. 3). The significant decrease of endocytic activity of iDC cultivated in the the presence of BioBran was ascertained, when compared to control iDC, and 

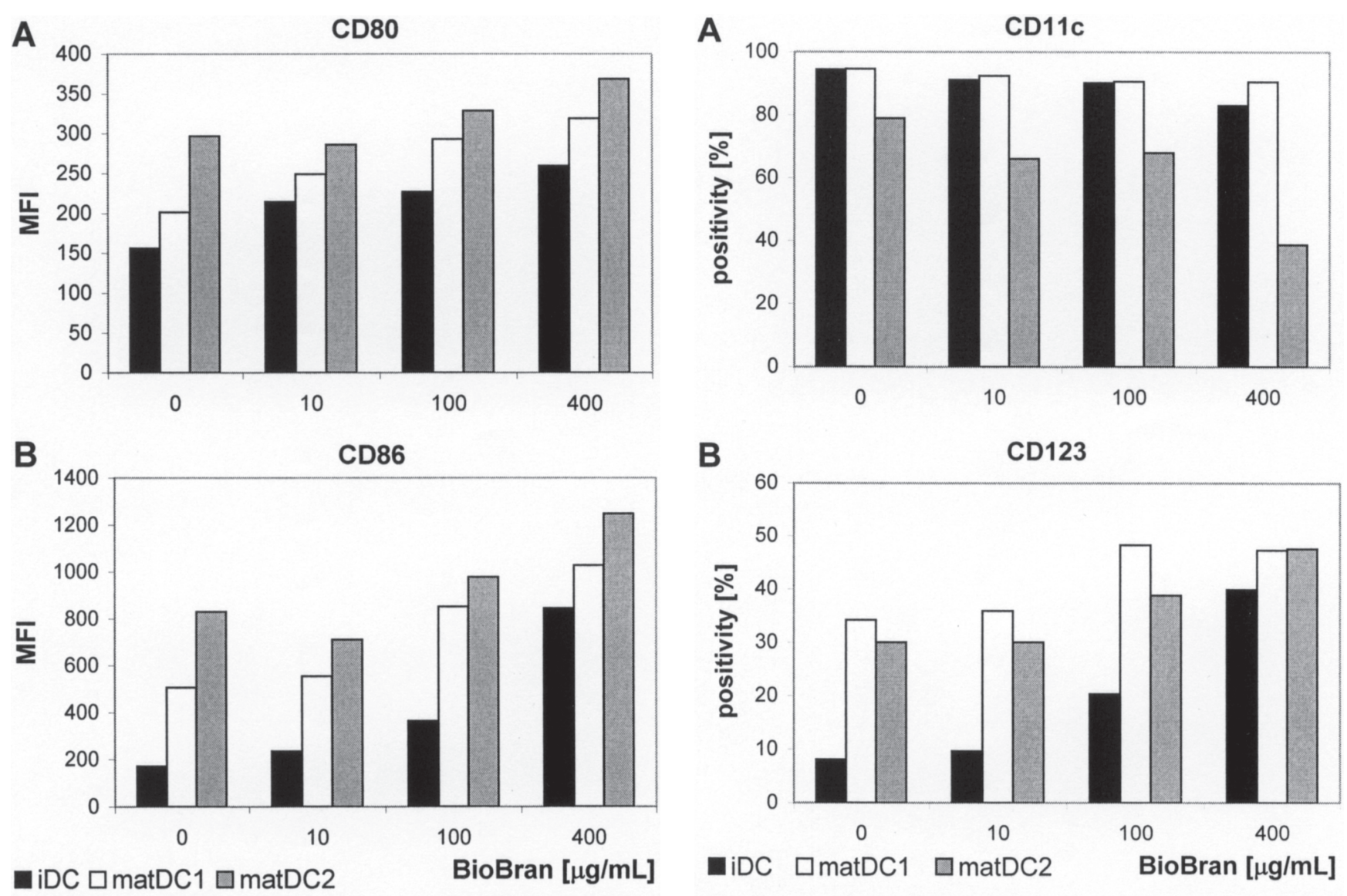

Figure 4. The effect of BioBran on the surface expression of costimulatory molecules. The cell surface expression of CD80 and CD86 markers was measured by flow cytometry on iDC, matDC1 and matDC2 populations cultured in the presence of relevant cytokine combination without (control cells) or with addition of BioBran at concentrations indicated. Results are shown as mean fluorescent intensities (MFI) and are representative of three independent experiments.

this effect of BioBran was dose-dependent (27.7\% for BioBran $100 \mu \mathrm{g} / \mathrm{ml}, 17.7 \%$ for BioBran $400 \mu \mathrm{g} / \mathrm{ml}$, and $14.4 \%$ of dextran-positive cells for BioBran $1000 \mu \mathrm{g} / \mathrm{ml}$; Fig. 3). Similar results were observed with monocytes, when $100 \mu \mathrm{g} / \mathrm{ml}$ of BioBran decreased the percentage of FITC-dextran positive cells to $12.7 \%$.

BioBran increases surface expression of costimulatory molecules $C D 80$ and $C D 86$. In order to assess the effect of BioBran on the expression of costimulatory molecules CD80 and CD86, Mo-derived iDC were treated for 2 days with BioBran $(10,100,400 \mu \mathrm{g} / \mathrm{ml})$ or matured with CMM 1 or CMM2 in the presence of BioBran. The cell surface expression of CD80 and CD86 on the iDC, matDC1 and matDC2 were studied by flow cytometric analysis on HLA-DR positively gated cells (Fig. 4). Control matDC2 matured in the presence of LPS and IFN- $\gamma$ showed the highest levels of CD80 (MFI 296), compared with control matDC1 matured in the

presence of IL-1 $\beta$, IL-6, and TNF- $\alpha$ (MFI 201) or immature DC (MFI 156). Similarly, matDC2 expressed the highest levels of CD86 (MFI 829) compared with matDC1 (MFI 506) and iDC (MFI 170). BioBran increased the mean of fluorescence intensity of costimulatory molecules CD80 and CD86 in dose dependent manner on all DC subsets. In the presence of BioBran in concentration of $400 \mu \mathrm{g} / \mathrm{ml}$, the MFI of CD80 increased to 368 for $\mathrm{mDC}, 319$ for matDC1 and 260 for iDC. Even more BioBran $(400 \mu \mathrm{g} / \mathrm{ml})$ increased markedly CD86 antigen (MFI 1248 for matDC2, 1027 for matDC1 and 845 for iDC).

BioBran up-regulates interleukin 3 receptor $\alpha$ chain CD123. The up-regulation of CD123, the alpha subunit of the human interleukin-3 receptor abundantly expressed on plasmacytoid DC, and down-regulation of the adhesion molecule CD11c on the cell surface of matDC2 cells in the presence of BioBran was observed (Fig. 5). BioBran $400 \mu \mathrm{g} / \mathrm{ml}$ induced 2-fold de- 
crease of CD11c positivity on matDC1 compared with control cells (from $78.9 \%$ to $38.7 \%$ ). In contrast, BioBran increased CD123 positivity on $\mathrm{iDC}, \mathrm{mDC}$ and matDC1 in dose dependent manner. The most evident effect of BioBran was seen on iDC, where BioBran $400 \mu \mathrm{g} / \mathrm{ml}$ induced 5-fold increase of CD123 positivity compared with control cells (from $8.1 \%$ to $39.9 \%$ ). In the presence of BioBran, iDC, matDC1 and matDC2 subsets expressed higher level of CD123 and lower level of CD11c cell surface antigens, the phenotype represented by $\mathrm{CD}_{11} \mathrm{c}^{\mathrm{dim}} \mathrm{CD} 123^{\text {bright }}$ plasmacytoid DC population.

\section{Discussion}

BioBran is a food supplement derived from rice bran hemicellulose enzymatically treated with multiple hydrolyzing enzymes obtained from Lentinus edodes mycelia (Shitake mushrooms). In previous studies BioBran showed immunomodulatory effects on innate (NK cells, macrophages) as well as on adaptive (T and B lymphocytes) immune system cells [11]. BioBran was shown to be a potent enhancer of NK cell activity $[8,9,12]$, accompanied by increased secretion of TNF- $\alpha$ and IFN- $\gamma$. BioBran also increased macrophage phagocytosis by induced production of TNF- $\alpha$, IL- 6 and nitric oxide $[13,14]$. DC are the most efficient antigen-presenting cells and play a central role in generating and regulating antitumor immunity. After capturing tumor antigens iDC undergo phenotypical and functional changes and differentiate into matDC. There is an intimate crosstalk between DC and NK cells at the beginning of immune response against viruses and tumor cells [15]. Based on aforementioned effects of BioBran on NK activity we wanted to delineate its possible role on DC maturation in vitro.

We have applied two maturation procedures that are used for preparation of DC from peripheral blood monocytes. Flow cytometric analysis of cell surface antigen expression as well as the test of dextran beads uptake confirmed the effective production of both immature and mature DC. BioBran demonstrated the concentration-dependent diminish of CD14 and augmentation of CD83 differentiation markers in matDC1 and iDC cells, respectively. In addition, the decrease of dextran beads uptake and the upregulation of costimulatory molecules expression supported the shift towards the more mature DC phenotype induced by BioBran.

The cytokine pattern released from tumor cells and tumor stromal compartments composed of fibroblasts, endothelial cells and infiltrating immune cells cooperatively affects DC activation. Tumor infiltration by CTL or by T, NK, and NKT cells is associated with favorable prognosis in different neoplasias such as melanoma, colon, and ovarian carcinomas [16-18]. On the other side unefficient activation of iDC in pancreatic carcinoma [19], induction of T-cell anergy mediated by APC cells [20], or altered DC infiltration pattern along the colorectal adenoma-carcinoma sequence [21] are examples how tumors avoid immune recognition.

BioBran treatment induced the concentration-dependent augmentation of CD123 antigen expression in both immature and mature DC, while decrease of CD11c expression was observed in matDC2 cells only. Thus the phenotype of CMM2matured DC resembles the typical one of plasmacytoid DC. Recently, the production of mDC from CD34+ cells, that express markers of both myeloid and plasmacytoid DC was described [22]. Similarly, the coexpression of CD123 and CD11c antigens on monocyte-derived DC as well as low FITClabeled dextran uptake due to maturation was observed [23]. This is in accordance to our results obtained by combination of BioBran with CMM1. On the other side, the cord bloodderived subset of CD123+ mDC with high uptake of FITC-labeled dextran that correlated with immature phenotype and with a significant tumor-inhibiting activity was described [24].

Until today, the mechanism in which BioBran alone or in combination with maturation mixes affects the expression of costimulatory and differentiation markers remains unclear. In addition to immunomodulatory effects, BioBran enhanced yeast-induced apoptosis of MCF-7 cells [25] and sensitized human $\mathrm{T}$ cell leukemia cells to death receptors CD95- induced apoptosis [26].

We assume that BioBran as an effective biological response modifier may stimulate DC maturation in vivo also. DC are central regulators of immunity bridging innate and adaptive immune responses, their presence in tumor microenvironment correlates with survival [27] although their numbers and functionality is affected in cancer patients [28, 29].

Possible beneficial effect of BioBran in future scenario in which the removal of inhibitory signals in the tumor microenvironment in combination with other therapeutic strategies could be successful in overcoming immunological insufficiency.

The authors gratefully acknowledge expert technical assistance by Mrs. Margita Sulikova and Mrs. Jana Chovancova. This work was supported by a commercial grant from Daiwa Pharmaceutical Co. Ltd. We are grateful to all the blood donors for donating blood for this study.

\section{References}

[1] RESCHNER A, HUBERT P, DELVENNE P et al. Innate lymphocyte and dendritic cell cross-talk: a key factor in the regulation of the immune response. Clin Exp Immunol 2008; 152: 219-226.

[2] BANCHEREAU J, BRIERE F, CAUX C et al. Immunobiology of dendritic cells. Annu Rev Immunol 2000; 18: 767-811.

[3] STEINMAN RM, HAWIGER D, LIU K et al. Dendritic cell function in vivo during the steady state: a role in peripheral tolerance. Ann N Y Acad Sci 2003; 987: 15-25.

[4] LUTZ MB, SCHULER G. Immature, semi-mature and fully mature dendritic cells: which signals induce tolerance or immunity? Trends Immunol 2002; 23: 445-449.

[5] UENO H, KLECHEVSKY E, MORITA R et al. Dendritic cell subsets in health and disease. Immunol Rev 2007; 219: 118-142. 
[6] PERROT I, BLANCHARD D, FREYMOND N et al. Dendritic cells infiltrating human non-small cell lung cancer are blocked at immature stage. J Immunol 2007; 178: 2763 2769.

[7] RABINOVICH GA, GABRILOVICH D, SOTOMAYOR EM. Immunosuppressive strategies that are mediated by tumor cells. Annu Rev Immunol 2007; 25: 267-296.

[8] GHONEUM M, JEWETT A. Production of tumor necrosis factor-alpha and interferon-gamma from human peripheral blood lymphocytes by MGN-3, a modified arabinoxylan from rice bran, and its synergy with interleukin-2 in vitro. Cancer Detect Prev 2000; 24: 314-324.

[9] GHONEUM M, ABEDI S. Enhancement of natural killer cell activity of aged mice by modified arabinoxylan rice bran (MGN-3/Biobran). J Pharm Pharmacol 2004; 56: $1581-1588$.

[10] HOCHREIN H, O'KEEFFE M, LUFT T et al. Interleukin (IL)-4 is a major regulatory cytokine governing bioactive IL12 production by mouse and human dendritic cells. J Exp Med 2000; 192: 823-833.

[11] GHONEUM M. Anti-HIV activity in vitro of MGN-3, an activated arabinoxylane from rice bran. Biochem Biophys Res Commun 1998; 243: 25-29.

[12] KIM HY, KIM JH, YANG SB et al. A polysaccharide extracted from rice bran fermented with Lentinus edodes enhances natural killer cell activity and exhibits anticancer effects. J Med Food 2007; 10: 25-31.

[13] GHONEUM M, MATSUURA M. Augmentation of macrophage phagocytosis by modified arabinoxylan rice bran (MGN-3/biobran). Int J Immunopathol Pharmacol 2004; 17: 283-292.

[14] GHONEUM M, MATSUURA M, GOLLAPUDI S. Modified arabinoxylan rice bran (MGN3/Biobran) enhances intracellular killing of microbes by human phagocytic cells in vitro. Int J Immunopathol Pharmacol 2008; 21: 87-95.

[15] MORETTA L, FERLAZZO G, BOTTINO C et al. Effector and regulatory events during natural killer-dendritic cell interactions. Immunol Rev 2006; 214: 219-228.

[16] GALON J, COSTES A, SANCHEZ-CABO F et al. Type, density, and location of immune cells within human colorectal tumors predict clinical outcome. Science 2006; 313: 1960-1964.

[17] HAANEN JB, BAARS A, GOMEZ R et al. Melanoma-specific tumor-infiltrating lymphocytes but not circulating melanoma-specific $\mathrm{T}$ cells may predict survival in resected advanced-stage melanoma patients. Cancer Immunol Immunother 2006; 55: 451-458.
[18] ZHANG L, CONEJO-GARCIA JR, KATSAROS D et al. Intratumoral $\mathrm{T}$ cells, recurrence, and survival in epithelial ovarian cancer. N Engl J Med 2003; 348: 203-213.

[19] BELLONE G, CARBONE A, SMIRNE C et al. Cooperative induction of a tolerogenic dendritic cell phenotype by cytokines secreted by pancreatic carcinoma cells. J Immunol 2006; 177: 3448-3460.

[20] CUENCA A, CHENG F, WANG H et al. Extra-lymphatic solid tumor growth is not immunologically ignored and results in early induction of antigen-specific T-cell anergy: dominant role of cross-tolerance to tumor antigens. Cancer Res 2003; 63: 9007-9015.

[21] YUAN A, STEIGEN SE, GOLL R et al. Dendritic cell infiltration pattern along the colorectal adenoma-carcinoma sequence. APMIS 2008; 116: 445-456.

[22] WARD KA, STEWART LA, SCHWARER AP. CD34+-derived CD11c+ + + BDCA-1+ + CD123+ + DC: expansion of a phenotypically undescribed myeloid DC1 population for use in adoptive immunotherapy. Cytotherapy 2006; 8: 130140.

[23] HO CS, MUNSTER D, PYKE CM et al. Spontaneous generation and survival of blood dendritic cells in mononuclear cell culture without exogenous cytokines. Blood 2002; 99: 2897-2904.

[24] SHI J, IKEDA K, MAEDA Y et al. Identification of CD123(+) myeloid dendritic cells as an early-stage immature subset with strong tumoristatic potential. Cancer Lett 2008; 270:19-29.

[25] GHONEUM M, GOLLAPUDI S. Modified arabinoxylan rice bran (MGN-3/Biobran) enhances yeast-induced apoptosis in human breast cancer cells in vitro. Anticancer Res 2005; 25 : $859-870$.

[26] GHONEUM M, GOLLAPUDI S. Modified arabinoxylan rice bran (MGN-3/Biobran) sensitizes human T cell leukemia cells to death receptor (CD95)-induced apoptosis. Cancer Lett 2003; 201: 41-49.

[27] NAGORSEN D, VOIGT S, BERG E et al. Tumor-infiltrating macrophages and dendritic cells in human colorectal cancer: relation to local regulatory $\mathrm{T}$ cells, systemic T-cell response against tumor-associated antigens and survival. J Transl Med 2007; 5: 62-69.

[28] MARTIN-AYUSO M, ALMEIDA J, PEREZ-ANDRES M et al. Peripheral blood dendritic cell subsets from patients with monoclonal gammopathies show an abnormal distribution and are functionally impaired. Oncologist 2008; 13: 82-92.

[29] KOVAROVA L, BUCHLER T, POUR L et al. Dendritic cell counts and their subsets during treatment of multiple myeloma. Neoplasma 2007; 54: 297-303. 